03,05

\title{
Аномальное изменение размера спинового полярона в парамагнитной области температур в $\mathrm{La}_{1.2} \mathrm{Sr}_{1.8} \mathrm{Mn}_{2} \mathrm{O}_{7}$
}

\author{
(C) С.А. Гудин \\ Институт фризики металлов им. М.Н. Михеева УрО РАН, \\ Екатеринбург, Россия \\ E-mail: gudin@imp.uran.ru \\ Поступила в Редакцию 8 июля 2021 г. \\ В окончательной редакции 13 июля 2021 г. \\ Принята к публикации 16 июля 2021 г.
}

\begin{abstract}
Продолжены исследования магнитных и электрических свойств двойного перовскита $\mathrm{La}_{1.2} \mathrm{Sr}_{1.8} \mathrm{Mn}_{2} \mathrm{O}_{7}$, обладающего вблизи температуры Кюри величиной колоссального магнитосопротивления превышающей 1200. Показано, что наблюдаемое в $\mathrm{La}_{1.2} \mathrm{Sr}_{1.8} \mathrm{Mn}_{2} \mathrm{O}_{7}$ колоссальное магнитосопротивление хорошо описывается на основе „ориентационного“ и „спин-поляронного“ механизмов проводимости. Обнаружено, что в отсутствии магнитного поля, линейный размер спинового полярона, уменьшается с ростом температуры в ферромагнитной области, а при переходе манганита в парамагнитное состояние линейный размер начинает возрастать, достигая максимума при $180 \mathrm{~K}$. При температурах, превышающих $180 \mathrm{~K}$ аномальное температурное изменение размера спинового полярона исчезает. В отсутствии магнитного поля, обнаруженный пик на температурной кривой изменения размера спинового полярона максимален, с включением магнитного поля высота пика уменьшается. Предложены механизмы, объясняющие такое аномальное температурное поведение размера спинового полярона.
\end{abstract}

Ключевые слова: колоссальное магнитосопротивление, спиновый полярон, слоистые манганиты, механизмы проводимости.

DOI: $10.21883 /$ FTT.2021.12.51653.31s

\section{1. Введение}

Открытие колоссального магнитосопротивления (КМС) [1,2], а именно эффекта, в котором удельное сопротивление образца при включении магнитного поля изменяется в десятки и более раз, заставило задуматься о механизмах проводимости так сильно зависящих от приложенного внешнего магнитного поля. Приложенное магнитное поле не изменяет проекцию скорости частиц на направление приложенного электрического поля, но благодаря силе Лоренца закручивает их траектории в плоскости, перпендикулярной приложенному магнитному полю. В результате за время между двумя столкновениями (время свободного пробега) частица пройдет путь вдоль направления приложенного электрического поля меньший, чем в отсутствие магнитного поля, т.е. сопротивление в магнитном поле из-за силы Лоренца должно увеличиваться. В манганитах, обладающих эффектом КМС, сопротивление в магнитном поле обычно уменьшается. До сих пор не создана последовательная микроскопическая теория, адекватно описывающая свойства таких допированных манганитов со структурой перовскита. Однако было установлено [3-7], что возникновение КМС в допированных манганитах связано с образованием неоднородных зарядовых и спиновых состояний (редкая земля при допировании замещается на металл, имеющий другую валентность, что и приводит к образованию зарядовых неоднородностей). Основное объяснение такой большой величины магнитосопротив- ления (МС), предполагает существование в допированных манганитах фазового расслоения на низко- и высокорезестивную фазы, размеры которых увеличиваются с ростом величины приложенного магнитного поля, увеличение размера может привести к соприкосновению низкоомных включений, что, в свою очередь, приводит к сильному уменьшению сопротивления вблизи порога протекания. Образование зарядовых неоднородностей приводит в манганитах к возникновению поляронов, но так как эти поляроны имеют некоторый объем, а манганиты являются ферро- или антиферромагнетиками, то на занятый поляроном объем приходится часть электронной спиновой плотности. Т.е. такой полярон имеет некий собственный магнитный момент, поэтому говорят о магнитных или спиновых поляронах (ферронах) [8,9]. Механизм магнитосопротивления, в котором сопротивление зависит от размера магнитных неоднородностей назван „размерным“ [10] или „спин-поляронным“ [11].

\section{2. Описание проводимости в магнитно-фазово-расслоенном манганите}

При протекании тока через магнитно-фазово-расслоенный манганит носитель тока переходит из одной магнитоупорядоченной области в другую. В отсутствие магнитного поля направления магнитных моментов этих областей разориентированы. С ростом величины прило- 
женного внешнего магнитного поля направления магнитных моментов ферромагнитноупорядоченных областей начинают ориентироваться по полю, полностью ориентируясь вдоль него в больших полях. Если длина свободного пробега носителя тока без переворота спинового магнитного момента больше расстояния между магнитоупорядоченными областями, то удельное сопротивление будет зависеть (как и в случае металлических сверхрешеток обладающих гигантским магнитосопротивлением (ГМС) [12,13]) от направлений магнитного упорядочения ферромагнитноупорядоченных областей, так называемый „ориентационный“ механизм проводимости $[14,15]$. Т.е. этот механизм проводимости, определяющий ГМС в магнитных металлических сверхрешетках, может вносить вклад и в КМС в магнитнофазово-расслоенных манганитах. Таким образом, проводимость $\sigma$ в монокристаллических допированных манганитах, обладающих КМС, определяется „ориентационным“ и „размерным“ механизмами $(\theta-$ и $r$-):

$$
\sigma(H)=\sigma_{r}(H)+\sigma_{\theta}(H),
$$

где $H-$ внешнее магнитное поле.

Для каждого конкретного значения температуры ориентационный механизм в магнитных полях больших поля насыщения вносит в проводимость постоянный вклад (магнитные моменты в полях превышающие поле магнитного насыщения не меняют своей ориентации, так как уже выстроены вдоль направления приложенного магнитного поля).

$$
\sigma_{\theta}\left(H>H_{\text {sat }}\right)=P_{1}(T)
$$

здесь $P_{1}=$ const для конкретного значения $T$.

В $[8,16]$ была описана спин-поляронная удельная проводимости $\sigma_{r}$ :

$$
\sigma_{r}(T, H)=B \sigma_{0} \frac{\omega_{0}}{T} \exp \left(-\frac{A(H)}{2 T}\right),
$$

где $T$ - температура, $\sigma_{0}-$ остаточная проводимость образца, $\omega_{0}$ - характерная магнонная частота, $B$ размерный множитель. $A(H)$ - энергетический барьер определяющийся кулоновским отталкиванием между совершающим прыжок электроном с зарядом $e$ и поляроном с зарядом $q$ :

$$
A(H)=k \frac{e q}{R_{\mathrm{Pol}}(H)},
$$

$k=\frac{1}{4 \pi \varepsilon \varepsilon_{0}}, \varepsilon-$ диэлектрическая проницаемость спинового полярона, $\varepsilon_{0}$ - электрическая постоянная, $R_{\mathrm{Pol}}-$ радиус полярона (в отсутствии магнитного поля, полярон имеет сферическую форму). С увеличением магнитного поля $H$ размер полярона $R_{\text {Рог }}$ вдоль направления приложения поля растет

$$
R_{\mathrm{Pol}}(T, H)=x(T)+y(T) H,
$$

$(x(T)$ и $y(T)$ принимают только положительные значения), форма полярона искажается, вытягиваясь вдоль приложенного поля, и $R_{\text {Pol }}$ становится равной половине размера полярона вдоль направления приложенного магнитного поля. В дальнейшем для краткости величину $R_{\text {Pol }}$ будем называть радиусом полярона.

Введя обозначения

$$
P_{2}(T)=B \sigma_{0} \frac{\omega_{0}}{T}, \quad P_{3}(T)=-\frac{k e q}{2 T y(T)}
$$

и

$$
P_{4}(T)=x(T) / y(T)
$$

получим формулу для проводимости для $H>H_{s a t}$ в параметрическом виде

$$
\sigma(H)=P_{1}+P_{2} \exp \left(\frac{-P_{3}}{P_{4}+H}\right) .
$$

Как следует из (6), параметр $P_{2}(T)$ обратно пропорционален температуре и для его вычисления используем полученные ранее для температуры $T=75 \mathrm{~K}$ значения этого параметра $P_{2}(T=75 \mathrm{~K})=(174 \pm 3) \mathrm{S} / \mathrm{cm}[11]$. Используя метод разделения вкладов в проводимость от „ориентационного“ и „спин-поляронного“ механизмов рассеяния $[10,15,17]$ для каждой конкретной температуры проводится расчет удельной проводимости образца.

\section{3. Исследования магнитных и электрических свойств двойного перовскита $\mathrm{La}_{1.2} \mathrm{Sr}_{1.8} \mathrm{Mn}_{2} \mathrm{O}_{7}$}

В настоящей работе продолжены исследования магнитных и электрических свойств двойного перовскита $\mathrm{La}_{1.2} \mathrm{Sr}_{1.8} \mathrm{Mn}_{2} \mathrm{O}_{7} \quad[11,18,19]$, обладающего вблизи температуры Кюри величиной колоссального магнитосопротивления превышающей 1200. Экспериментальные данные по температурным и магнитным изменениям сопротивления монокристаллического $\mathrm{La}_{1.2} \mathrm{Sr}_{1.8} \mathrm{Mn}_{2} \mathrm{O}_{7}$, используемые в данной работе и представленные на рис. 1 , взяты из работы [11].

Было обнаружено, что в парамагнитной области температур в отсутствие магнитного поля размер полярона с ростом температуры ведет себя не обычно, но для более глубокого понимания происходящих в этой области температур процессов расчетных данных не хватало. Возник вопрос, связано ли обнаруженное отклонение от монотонного спада в области выше температуры Кюри с погрешностями расчета, и таким образом оно является флуктуационным биением, обусловленным тем, что для каждой точки на графике „температура относительный размер спинового полярона“ необходимо было проводить отдельный расчет, или обнаруженная аномалия связана с физическими процессами? В работе проведены дополнительные расчеты, дающие возможность однозначно ответить на этот вопрос. 


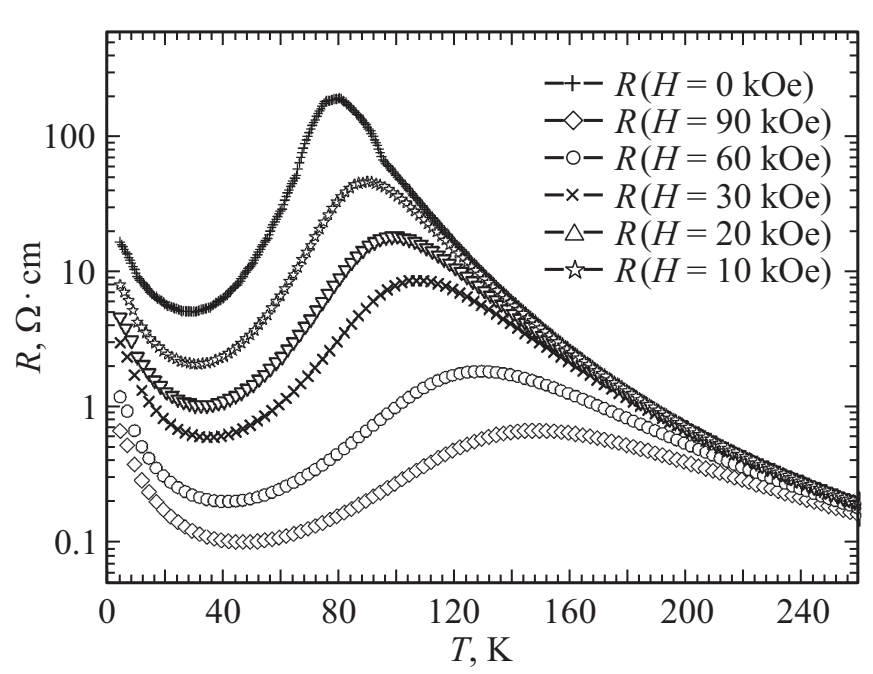

Рис. 1. Электросопротивление в зависимости от температуры $T$ при значениях поля $H$ : $0,10,20,30,60,90 \mathrm{kOe}$.
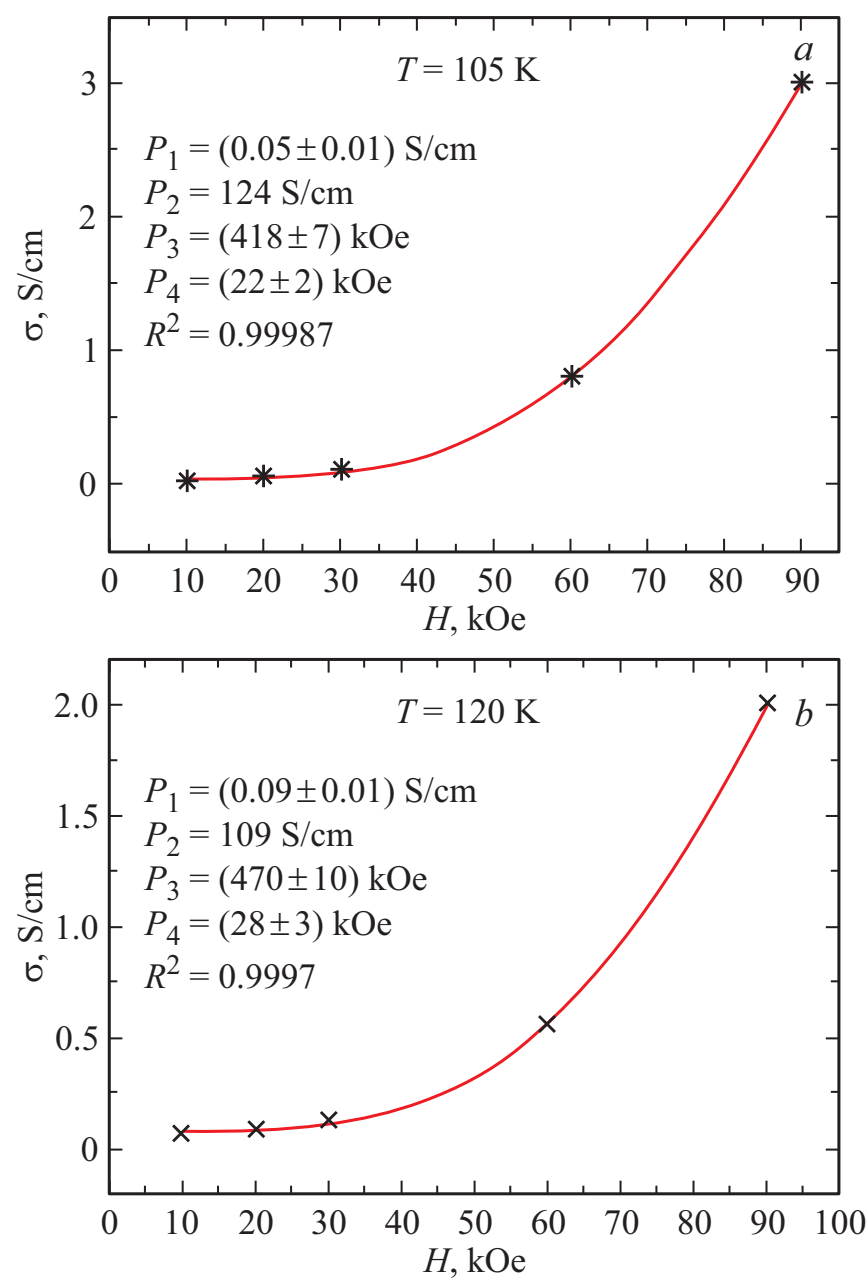

Рис. 2. Удельная проводимость в магнитном поле $H$ : $(a)$ для температуры $105 \mathrm{~K},(b)$ для температуры $120 \mathrm{~K}$. Точки экспериментальные значения, сплошные линии - расчет. На вставках полученные значения параметров (2) и (6), $R^{2}-$ коэффициент детерминации.

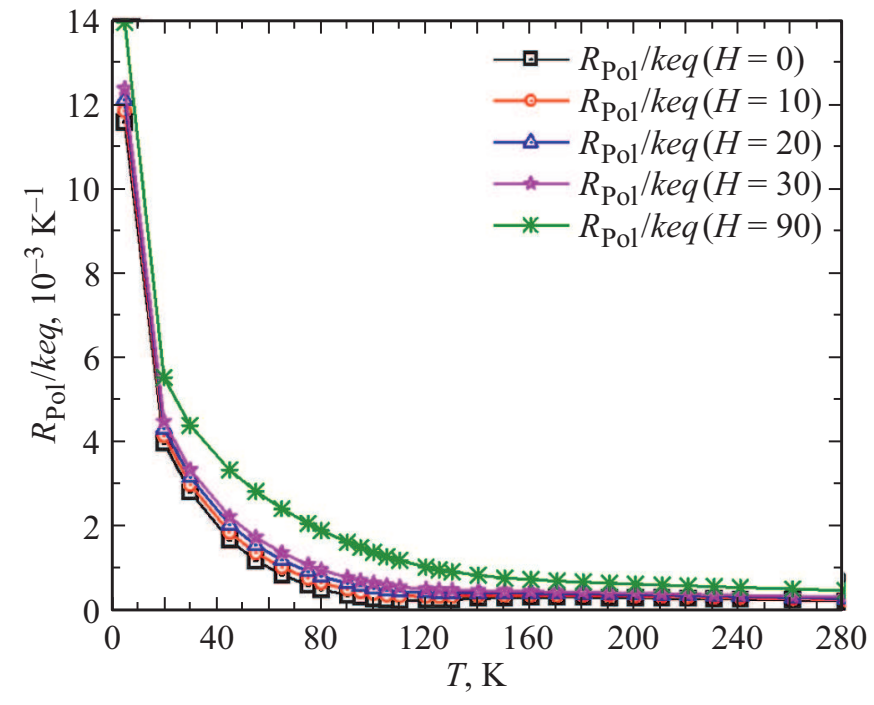

Рис. 3. Изменение радиуса полярона, отнесенного к константе $k e q$, в зависимости от температуры $T$ при значениях поля $H$ : $0,10,20,30,90 \mathrm{kOe;} \mathrm{точки} \mathrm{обозначены} \mathrm{квадратами,} \mathrm{кружка-}$ ми, треугольниками, звездочками, снежинками, соответственно (обозначения для разных температур приведены на вставках).

На рис. 2 приведена удельная проводимость $\mathrm{La}_{1.2} \mathrm{Sr}_{1.8} \mathrm{Mn}_{2} \mathrm{O}_{7}$ в магнитном поле $H:(a)$ - для температуры $105 \mathrm{~K},(b)$ - для температуры $120 \mathrm{~K}$. Точки - экспериментальные значения, сплошные линии - расчет. На вставках полученные значения параметров (2) и (6). $R^{2}$ - коэффициент детерминации, характеризующий на сколько хорошо коррелируют расчетные и экспериментальные данные. Значение коэффициента детерминации 1 означает функциональную зависимость между переменными. Вычисленные кривые удельной проводимости проходят через экспериментальные точки с достаточно большой точностью рис. 2. Получено, что коэффициент детерминации превышает значение 0.9996 для всех температур при которых производились расчеты (для $T=105 \mathrm{~K} R^{2}=0.999987$, а для $T=120 \mathrm{~K} R^{2}=0.9997$ см. вставки на рис. 2, $a$ и $b$ ). Значение коэффициентов детерминации, близких к 1 , указывает на то, что отклонение экспериментальных точек от расчетных кривых минимально.

Согласно (5) и (6) запишем радиус полярона в единицах keq:

$$
R_{\mathrm{Pol}}(T, H) / k e q=\frac{\left(P_{4}+H\right)}{2 P_{3} T} .
$$

На рис. 3 представлено изменение радиуса полярона, отнесенного к константе keq, в зависимости от изменения температуры $T$ при значениях поля $H$ : 0,10 , 20, 30, $90 \mathrm{kOe}$, расчетные значения обозначены квадратами, кружками, треугольниками, звездочками, снежинками соответственно. Для наглядности тенденций, расчетные точки для каждого значения магнитного поля соединены отрезками. С ростом температуры размер полярона уменьшается. Однако в области выше $110 \mathrm{~K}$ 


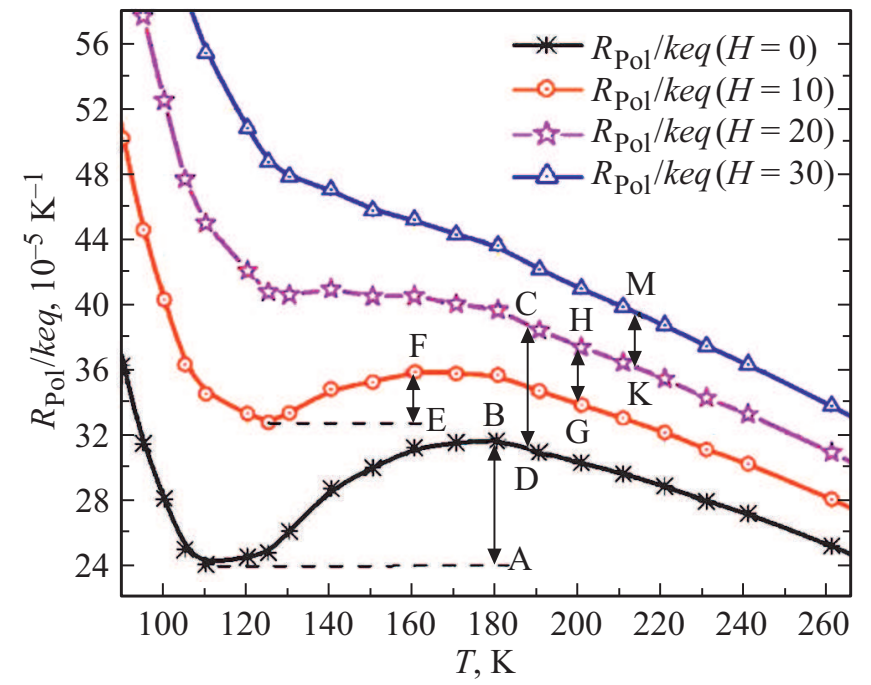

Рис. 4. Размер полярона в относительных единицах в зависимости от температуры $T$ при значениях поля $H$ : $0 \mathrm{kOe}$ (снежинки), $10 \mathrm{kOe}$ (кружки), $20 \mathrm{kOe}$ (звездочки), $30 \mathrm{kOe}$ (треугольники) (обозначения для разных температур приведены на вставках).

для полей меньших $90 \mathrm{kOe}$ обнаружено, как было ранее упомянуто, аномальное поведение изменения размера спинового полярона (см. рис. 4). В отсутствии магнитного поля, линейный размер спинового полярона, уменьшается с ростом температуры в ферромагнитной области, а при переходе манганита в парамагнитное состояние линейный размер начинает возрастать, достигая максимума при $180 \mathrm{~K}$. При температурах, превышающих $180 \mathrm{~K}$ аномальное температурное изменение размера спинового полярона исчезает. В отсутствии магнитного поля, обнаруженные изменения („горб“ на кривой) размера полярона максимален, с включением магнитного поля высота „горба“ уменьшается. В поле $20 \mathrm{kOe} \mathrm{„горб“} \mathrm{полностью} \mathrm{исчезает,} \mathrm{переходя} \mathrm{в} \mathrm{точку}$ перегиба на температурной кривой относительного размера спинового полярона. В магнитных полях больше $30 \mathrm{kOe}$ аномальное температурное поведение размера полярона полностью пропадает.

На рис. 4 представлено изменение радиуса полярона, отнесенного к константе keq, в зависимости от температуры $T$ для диапазона температур от 90 до $260 \mathrm{~K}$ в магнитных полях $H$ : 0, 10, 20, 30 kOe.

Включение магнитного поля увеличивает размер спинового полярона. Аномальное увеличение размера спинового полярона при температурах выше $110 \mathrm{~K}$ („горб“ на нижней кривой рис. 4) на величину отрезка $[\mathrm{AB}]$ практически эквивалентно энергетическому воздействию магнитным полем $20 \mathrm{kOe}$ (длины отрезков $[\mathrm{AB}]$ и $[\mathrm{CD}]$ равны). В магнитном поле $10 \mathrm{kOe}$ размер спинового полярона претерпевает изменения аналогичные изменениям в отсутствии магнитного поля, но увеличение размера начинается с температуры $125 \mathrm{~K}$ и высота „горба“ (отрезок $[\mathrm{FE}]$ ) на графике меньше.
Аномальное увеличение размера практически эквивалентно энергетическому воздействию магнитным полем $10 \mathrm{kOe}$ (длины отрезков $[\mathrm{FE}]$ и $[\mathrm{HG}]$ равны). Увеличение магнитного поля с 20 до $30 \mathrm{kOе}$ приводит к увеличению размера спинового полярона сопоставимому с увеличением размера при изменении магнитного поля с 10 до $20 \mathrm{kOe}$ (длины отрезков $[\mathrm{KM}]$ и $[\mathrm{HG}]$ равны), горб полностью пропадает.

Спиновые поляроны образовываются из-за ферромагнитных зарядовых неоднородностей, создаваемых в манганите допированием элементом другой валентности. Образованию квазичастиц препятствует температура, стремящаяся их разрушить. Магнитное поле, воздействуя на магнитный момент спинового полярона, действует против разупорядочивающего воздействия температурных колебаний решетки, что приводит к росту размера полярона при включении магнитного поля. Но для ферромагнитой области температур на спиновый полярон действует среднее магнитное поле, стремящееся коллективизировать обособленные магнитные моменты поляронов. В результате размер полярона определяется минимизацией его свободной энергии поверхности. При переходе в парамагнитную область с дальнейшим ростом температуры препятствующее образованию полярона воздействие среднего магнитного поля исчезает, что приводит к увеличению свободной энергии поверхности, и как результат к увеличению размера спинового полярона. Дальнейший рост температуры компенсирует эффект от увеличения свободной энергии и после достижения некоторой температуры аномальный рост размера полярона исчезает, сменяясь тенденцией уменьшения размера.

\section{4. Заключение}

В работе продолжены исследования магнитных и электрических свойств двойного перовскита $\mathrm{La}_{1.2} \mathrm{Sr}_{1.8} \mathrm{Mn}_{2} \mathrm{O}_{7}$, обладающего вблизи температуры Кюри величиной колоссального магнитосопротивления превышающей 1200. Показано, что магнитосопротивление в рассматриваемом соединении определяется изменением размеров и направлений магнитных моментов магнитных неоднородностей (спиновых поляронов), - „спин-поляронный“ и „ориентационный“ механизмы соответственно. Удельная проводимость определяется прыжковой проводимостью носителей с одного спинового (ферромагнитного) полярона на соседний. Наблюдаемое в $\mathrm{La}_{1.2} \mathrm{Sr}_{1.8} \mathrm{Mn}_{2} \mathrm{O}_{7}$ колоссальное магнитосопротивление хорошо описывается на основе „ориентационного“ и „спин-поляронного“ механизмов проводимости. В работе обнаружено, что в отсутствии магнитного поля, линейный размер спинового полярона, уменьшается с ростом температуры в ферромагнитной области, а при переходе манганита в парамагнитное состояние линейный размер начинает возрастать, достигая максимума при $180 \mathrm{~K}$. При температурах превышающих $180 \mathrm{~K}$ аномальное температур- 
ное изменение размера спинового полярона исчезает. В отсутствии магнитного поля, обнаруженный пик на температурной кривой изменения размера спинового полярона максимален, с включением магнитного поля высота пика уменьшается. В поле $20 \mathrm{kOе}$ пик полностью исчезает, переходя в точку перегиба на температурной кривой размера спинового полярона. В магнитных полях больше $30 \mathrm{kOe}$ аномальное температурное поведение размера полярона полностью пропадает. Предложены механизмы, объясняющие такое аномальное температурное поведение размера спинового полярона.

\section{Благодарности}

Авторы благодарят К.И. Кугеля и Н.И. Солина за ценные замечания, сделанные при обсуждении результатов этой работы.

\section{Финансирование работы}

Работа выполнена в рамках государственного задания МИНОБРНАУКИ „Квант“ № АААА-А18118020190095-4 при частичной поддержке РФФИ (проект № 19-02-01000).

\section{Конфликт интересов}

Автор заявляет, что у него нет конфликта интересов.

\section{Список литературы}

[1] Э.Л. Нагаев. УФН 166, 8, 833, (1996).

[2] Y. Tokura, Y. Tomioko. JMMM 200, 1 (1999).

[3] Y. Coey, M. Viret, S. von Molnor. Adv. Phys. 48, 167 (1999).

[4] E. Dagotto. Nanoscale Phase Separation and Colossal Magnetoresistance. Springer-Verlag, Berlin (2002). p. 452.

[5] M.B. Salamon, M. Jaime. Rev. Mod. Phys. 73, 583 (2001).

[6] R. von Helmholtz, J. Wecker, B. Holzapfel, L. Schultz, K. Samwer. Phys. Rev. Lett. 71, 2331 (1993).

[7] S. Jin, T.H. Tiefel, M. Mc Cormack, R.A. Fastnacht, R. Ramesh, L.M. Chen. Science 264, 413 (1994).

[8] A.L. Rakhmanov, K.I. Kugel', Ya.M. Blanter, M.Yu. Kagan. J. Phys. Rev. B 63, 174424 (2001).

[9] К.И. Кугель, А.Л. Рахманов, А.О. Сбойчаков, М.Ю. Каган, И.В. Бродский, А.В. Клапцов. ЖЭТФ 125, 648 (2004).

[10] М.И. Куркин, Э.А. Нейфельд, А.В. Королев, Н.А. Угрюмова, С.А. Гудин, Н.Н. Гапонцева. ЖЭТФ 55, 5, 896 (2013).

[11] С.А. Гудин, Н.И. Солин, Н.Н. Гапонцева. ФТТ 60, 6, 1067 (2018). [S.A. Gudin, N.I. Solin, N.N. Gapontseva. Phys. Solid State 60, 6, 1078 (2018).]

[12] M. Zabel. J. Phys. Condens. Matter 11, 9303 (1999).

[13] D.T. Pierce, J. Unguris, R.J. Celotta, M.D. Stiles. JMMM 200, 290 (1999).

[14] М.И. Куркин, Э.А. Нейфельд, А.В. Королев, Н.А. Угрюмова, С.А. Гудин, Н.Н. Гапонцева. ЖЭТФ 143, 5, 948 (2013). [M.I. Kurkin, E.A. Neifel'd, A.V. Korolev, N.A. Ugryumova, S.A. Gudin, N.N. Gapontseva. JETP 116, 5, 823 (2013)].
[15] С.А. Гудин, М.И. Куркин, Э.А. Нейфельд, А.В. Королев, Н.А. Угрюмова, Н.Н. Гапонцева. ЖЭТФ 148, 5, 1005 (2015). [S.A. Gudin, M.I. Kurkin, E.A. Neifel'd, A.V. Korolev, N.A. Ugryumova, N.N. Gapontseva. JETP 121, 5, 878 (2015)].

[16] М.Ю. Каган, К.И. Кугель. УФН 171, 6, 577 (2001). [M.Yu. Kagan, K.I. Kugel'. Phys.-Usp. 44, 6, 553 (2001).]

[17] С.А. Гудин, Н.Н. Гапонцева, Э.А. Нейфельд, А.В. Королев, Н.А. Угрюмова. Изв. РАН. Сер. Физ. 78, 9, 1142 (2014). [S.A. Gudin, N.N. Gapontseva, E.A. Neifel'd, A.V. Korolev, N.A. Ugryumova. Bull. Russ. Acad. Sci.: Phys. 78, 9, 900 (2014)].

[18] С.А. Гудин, Н.И. Солин. ЖЭТФ 157, 4, 648 (2020). [S.A. Gudin, N.I. Solin. J. Exp. Theor. Phys. 130, 4, 543 (2020)].

[19] С.А. Гудин, Н.И. Солин. ФТТ 62, 5, 669 (2020). [S.A. Gudin, N.I. Solin. Phys. Solid State 62, 5, 756 (2020)].

Редактор Ю.Э. Китаев 